\title{
Complicações da terapia anticoagulante com warfarina em pacientes com doença vascular periférica: estudo coorte prospectivo
}

\author{
Complications of anticoagulant therapy with warfarin in patients \\ with peripheral vascular disease: a cohort prospective study \\ Fernada C ardoso Santos ${ }^{1}$, Francisco H umberto de Abreu M affei ${ }^{2}$, \\ Lidia Raquel de Carvalho ${ }^{3}$, Izolete Aparecida Tomazini-Santos', M ariangela Gianini ${ }^{5}$, \\ Marcone Lima Sobreira ${ }^{6}$, Paulo Eduardo Arbex 7 , Ana Paula M órbio ${ }^{8}$
}

\section{Resumo}

O bjetivo: Estudar prospectivamente a freqüência de complicações em pacientes tratados com warfarina eacompanhados no Ambulatório de Anticoagulação da Faculdade de M edicina de Botucatu da U niversidade Estadual Paulista.

M étodos: Pacientes sorteados entre os agendados para consulta de junho de 2002 a fevereiro de 2004. Na primeira consulta, foi preenchida ficha com dados de identificação e clínicos. A cada retorno, ou quando o paciente procurou o hospital por intercorrência, foi pre enchida ficha com a razão normatizada internacional, existência e tipo de intercorrência e condições de uso dos antagonistas da vitamina K.

Resultados: Foram acompanhados 136 pacientes (61 homens e 75 mulheres), 99 com tromboembolismo venoso e 37 com doença arterial; 59 pacientes eram de Botucatu, e 77, de outros municípios. Foram registradas 30 intercorrências: nove não relacionadas ao uso da warfarina e 21 complicações hemorrágicas (38,8 por 100 pacientes) ano). U ma hematêmese foi considerada grave (1,9 por 100 pacientes/ ano). As demais foram consideradas moderadas ou leves. $\mathrm{N}$ ão houve óbitos, hemorragia intracraniana ou necrose cutânea. A única associação significante foi da freqüência de hemorragia com nível médio de razão normatizada internacional.

C onclusão: N ossos resultados mostram a viabilidade desse tratamento em pacientes vasculares em nosso meio, mesmo em população de baixo nível socioeconômico, quando tratados em ambulatório especializado.

Palavras-chave: Anticoagulantes, warfarina, efeitos adversos, doenças vasculares, trombose venosa, embolia pulmonar.

\begin{abstract}
O bjective: To prospectively study the frequency of complications in patientstreated with warfarin followed at Botucatu M edical School.

M ethods: Patients randomly selected among those with appointments scheduled from June 2002 to February 2004. At the first appointment, a protocol was filled with identification and clinical data. At every return or when the patient went to the hospital due to clinical events, another form was filled with the international normalized ratio, existenceand type of clinical event and the conditions of use of vitamin $\mathrm{K}$ antagonists.
\end{abstract}

Results: A total of 136 patients (61 men and 75 women), were followed; 99 patients with venous thromboembolism and 37 with arterial disease; 59 were from Botucatu and 77 were from other municipalities. Thirty clinical events were registered: nine of them were not related to the use of warfarin and 21 were hemorrhagic complications (38.8 per 100 patients-year). O ne hematemesis was considered severe (1.9 per 100 patients-year). The others were considered moderate or mild. There were no deaths, intracranial hemorrhage or cutaneous necrosis. The only significant association was frequency of hemorrhage and average international normalized ratio level.

Conclusion: 0 ur results demonstrate the feasibility of this treatment in vascular patients in our country, even in socially and economically poor populations, when treated in specialized clinics.

Key words: Anticoagulants, warfarin, adverse effects, vascular diseases, venous thrombosis, pulmonary embolism.

\footnotetext{
1. Biomédica. Mestre em Bases Gerais da Cirurgia, Departamento de Cirurgia e Ortopedia, Faculdade de Medicina de Botucatu, Universidade Estadual Paulista (UNESP), Botucatu, SP

2. Coordenador, Grupo de Pesquisa em Trombose e Hemostasia, Instituto de Ensino e Pesquisa do Hospital Sírio Libanês, Botucatu, SP. Professor Programa de Pós-Graduação em Bases Gerais da Cirurgia, Faculdade de Medicina de Botucatu, UNESP, Botucatu, SP.

3. Doutora. Professora assistente, Departamento de Bioestatística, Instituto de Biociências de Botucatu, UNESP, Botucatu, SP.

4. Doutora. Bióloga-chefe, Laboratório de Hemostasia, Hemocentro, Faculdade de Medicina de Botucatu, UNESP, Botucatu, SP.

5. Doutora. Professora assistente, Departamento de Cirurgia e Ortopedia, Faculdade de Medicina de Botucatu, UNESP, Botucatu, SP.

6. Médico, Departamento de Cirurgia e Ortopedia, Faculdade de Medicina de Botucatu, UNESP, Botucatu, SP.

7. Mestre. Professor assistente, Departamento de Clínica Médica, Faculdade de Medicina de Botucatu, UNESP, Botucatu, SP.

8. Pós-graduanda, Programa de Pós-Graduação em Bases Gerais da Cirurgia, Faculdade de Medicina de Botucatu, UNESP, Botucatu, SP

Trabalho apresentado no 36 Congresso Brasileiro de Angiologia e Cirurgia Vascular, realizado em Porto Alegre, no período de 4 a 8 de setembro de 2005. Conflito de interesse: Fernanda Cardoso Santos recebeu bolsa de Mestrado da CAPES durante a realização deste trabalho.

Artigo submetido em 04.05.06, aceito em 11.09.06.
} 


\section{Introdução}

A utilidade dos antagonistas da vitamina K (AVK) foi estabelecida há mais de meio século para uma variedade de condições em trabalhos experimentais e ensaios clínicos, sendo hoje de uso universal. Vários estudos demonstraram que a utilização da anticoagulação no tromboembolismo venoso (TEV) diminuiu a recorrência tanto da trombose venosa profunda (TVP) como da embolia pulmonar $(E P)^{1,2}$. O s AVK são utilizados também para prevenir a embolia arterial sistêmica em pacientes com válvulas cardíacas ou fibrilação atrial ${ }^{1,3}, 0$ acidente vascular cerebral ${ }^{1,4}$, 0 infarto recorrente ${ }^{1}$ e a retrombose em pacientes submetidos à embolectomia ou trombectomia arterial, arterioplastias, endarterectomias e enxertos arteriais e venosos ${ }^{5}$.

O efeito terapêutico dos AVK varia de indivíduo para indivíduo, dependendo de fatores genéticos e ambientais, que influenciam sua absorção, farmacocinética e farmacodinâmica ${ }^{1}$.

0 s fatores genéticos incluem mutações no gene da enzima do citocromo P450 2C 9, quepodem reduzir as necessidades de warfarina em seus portadores ${ }^{6,7}$, e a mutação do gened o fator I $X$, quepodeaumentar o risco desangramento quando do uso dosAVK ${ }^{7,8}$. Há, ainda, uma resistência de origem genética à warfarina, que pode aumentar a necessidade de suas doses em até 20 vezes, para que se alcance 0 efeito anticoagulante desejado 9,10 .

M uitas medicações interagem com a warfarina potencializando ou reduzindo seu efeito ${ }^{1,8}$, e a atividade anticoagulante depende também do tipo de dieta utilizada pelo paciente, se mais ou menos rica em vitamina $\mathrm{K}^{11}$.

Esses fatos obrigam a um controle terapêutico rigoroso, com correção da dose do anticoagulante de acordo com o tempo de protrombina, devendo ser realizado com a utilização de tromboplastinas com índice de sensibilidade próximo ao do padrão internacional e expresso pela razão normatizada internacional $(\mathrm{RNI})^{12}$.

0 acompanhamento de pacientes em clínicas especializadas, principalmente em grandes hospitais comunitários, parece melhorar a eficácia e segurança do tratamento anticoagulante ${ }^{13-16}$.

As complicaçõesmaisimportantes efreqüentes que podem ocorrer com o uso dos AVK são as hemorragias ${ }^{1,8,17,18}$, que podem ter relação com 0 valor da $\mathrm{RN} \mathrm{I}^{19}$. Estudos prospectivos, que avaliaram complica- ções hemorrágicas da anticoagulação oral, mostraram freqüência de sangramentos considerados maiores, de 0,4 a $12 \%{ }^{18}$. Com menor freqüência, pode ocorrer a necrose depeleesubcutânea, queestá presenteem cerca de 0,01 a 0,1\% 18,20,21. 0 utras reações adversas incluem reações de hipersensibilidade, icterícia colestática, hepatite, vasculites, náuseas e vômitos, diarréia, alopecia, etc ${ }^{1,8,18}$. T ambém computado como complicação éo desenvolvimento deuma nova trombose ou retrombosena vigência do tratamento, o que pode ocorrer nos casos de TEV com uma freqüência de 3 a 15\%22.

0 tratamento de populações de baixo nível socioeconômico com AVK tem sido visto como um desafio, sendo mesmo contra-indicado por al guns autores. I sso se dá pela dificuldade de aderência desses pacientes ao tratamento, que seria agravada nos casos em que existe dificuldade geográfica de acesso ao local de atendimento, fatores que poderiam aumentar a freqüência de complicações ${ }^{23}$. 0 s trabal hos retrospectivos indicam a viabilidade desse tratamento em nosso meio 13,24,25. $\mathrm{N}$ ão temos, entretanto, conhecimento detrabalho prospectivo estudando as complicações dos anticoagulantes orais, especialmente em populações com essas características.

0 objetivo do presente trabalho foi estudar prospectivamente a freqüência de complicações durante 0 tratamento anticoagulante com AVK em pacientes portadores de doença vascular periférica, acompanhados no Ambulatório de Anticoagulação da Faculdade de M edicina de Botucatu (AAFM B) da U niversidade Estadual Paulista (UNESP), e compará-la com dados da literatura internacional, a fim de avaliar a viabilidade desse tratamento em nosso meio.

\section{Métodos}

Foram estudados 136 pacientes portadores de doença vascular, arterial ou venosa, acompanhados no AAFM B, entre julho de 2002 e fevereiro de 2004. Os casos de doença arterial incluíam pacientes com oclusão arterial aguda, tratados clinicamente ou submetidos à embolectomia ou trombectomia e mantidos perenemente anticoagulados, e pacientes submetidos a outras cirurgias arteriais e que, a critério do cirurgião, deveriam ser mantidosanticoagulados. 0 scasosvenososeram detrombosevenosae/ou EP confirmadosobjetivamente. 0 anticoagulante utilizado foi a warfarina (ou varfarina) sódica ( $\mathrm{M}$ arevan ${ }^{\circledR}$ ou Coumadin ${ }^{\circledR}$ ), que era fornecida para os pacientes em quantidade suficiente para durar até o retorno seguinte. 
U m dia antesou namanhã da realização das consultas, os pacientes foram sorteados entre os previamente agendados, excluindo osjá anteriormente colocadosno trabalho ou os que não aceitaram participar do trabaIho. $\mathrm{N}$ a primeira consulta, foi explicada ao paciente a finalidade do trabalho esolicitada a assinatura do termo de consentimento livre e esclarecido. A seguir, foi preenchida a ficha com dados de identificação e clínicos, incluindo indicação do tratamento anticoagulante e faixa de RN I desejada.

As consultas eram realizadas por um médico assistente ou residente de cirurgia vascular, de acordo com a rotina do serviço, sendo feita correção da dose do AVK quando a RN I estava acima ou abaixo da faixa desejada, em geral entre 2 e 3, utilizando-se vitamina $\mathrm{K}$ oral se julgado necessário. A R N I foi determinada utilizando 0 aparelho Thrombolyzer Compact 5 com reagenteSimplastin Excel $S^{\circledR}$ (Biomerieux).

$N$ ão houve interferência na conduta médica dos doentes, porém a pesquisadora responsável pelo preenchimento das fichas complementou a orientação e esclareceu dúvidas sobre o tratamento e/ou sobre a medicação usada, quando solicitado pelo paciente.

A faixa de RN I considerada como adequada para análise no presente estudo foi de 1,8 a 4,0. A cada retorno, ou quando o paciente procurou o hospital por qualquer intercorrência, foi preenchidaafichadeacompanhamento com a RN I, dose deAVK, outrosmedicamentos utilizados, alterações no hábito alimentar, ingestão de bebidas alcoólicas e existência e tipo de intercorrência.

Como intercorrências, foram registrados: qualquer alteração desaúdenão existenteno início do tratamento anticoagulante, sangramentos, sintomas de TVP e/ou $E P$, sintomas de nova oclusão arterial, sintomas de hipersensibilidade, necrose cutânea ou alopécia.

O sangramento foi classificado como:

- Grave: em caso de óbito, quando exigisse internação, suspensão definitiva da medicação, transfusão sangüínea ou quando ocorresse hemorragia cerebral, retroperitoneal ou gastrintestinal.

- M oderado: hematúria macroscópica, sangramento retal ou genital e epistaxe, desde que não levassem a situações classificadas como graves.

- Leve: equimose ou hematoma subcutâneo pequeno, epistaxe ou sangramento gengival, apenas referidos no retorno.
$\mathrm{N}$ os casos de pacientes que não comparecessem no dia previsto ou até 2 semanas após essa data, pretendiase entrar em contato tel efônico para verificar a causa da ausência e agendar nova consulta, providência que não se mostrou necessária.

0 tamanho da amostra foi definido considerandose percentuais deincidência de complicações citados na literatura e 0 número de pacientes atendidos no AAFM B durante 0 ano, utilizando-se 0 nível de $5 \%$ de significância euma precisão de $5 \%$, sendo determinado em, no mínimo, 136 pacientes. $A$ freqüência deeventos foi calculada para eventos/100 pacientes/ano. Para variáveis quantitativas, utilizou-se o teste t de Student, e para variáveis qualitativas, o teste qui-quadrado.

0 projeto foi aprovado pelo Comitê de Ética em Pesquisa da FM B.

\section{Resultados}

Foram estudados 136 pacientes, $75(55,1 \%)$ do sexo feminino e 61 (44,9\%) do sexo masculino. D estes, 59 eram da cidade de Botucatu, e 77, de outros municípios. A média deidadefoi de $56( \pm 12,4)$ anos (22-79 anos). 0 s pacientes foram divididos em três grupos, de acordo com a faixa etária, como mostra a T abela 1.

T abela 1 - Distribuição por faixa etária do grupo de pacientes com doença vascular periférica tratados com antagonista da vitamina $\mathrm{K}$

\begin{tabular}{lcc}
\hline Faixa etária & $\mathbf{n}$ & Percentagem \\
\hline $20-40$ anos & 13 & 9,6 \\
$40-60$ anos & 67 & 49,3 \\
$>60$ anos & 56 & 41,2 \\
Total & 136 & 100 \\
\hline
\end{tabular}

A distribuição do diagnóstico com relação ao sexo dos pacientes encontra-se na T abela 2.

D o total de pacientes, 78 foram tratados por um período igual ou maior que 6 meses, e 58, entre 3 e 6 meses. 0 número total de retornos foi de 2.170 , com uma média de 15,9 retornos por paciente. 
T abela 2 - D istribuição do grupo de pacientes com doença vascular periférica, tratados com antagonista da vitamina $\mathrm{K}$, segundo 0 sexo e a doença que levou ao tratamento anticoagulante

\begin{tabular}{lccc}
\hline D iagnóstico & \multicolumn{2}{c}{ Sexo } & \\
\cline { 2 - 3 } & Feminino & Masculino & T otal \\
\hline EP & $13(61,9 \%)$ & $8(38,1 \%)$ & 21 \\
D A & $15(40,5 \%)$ & $22(59,5 \%)$ & 37 \\
TVP & $47(60,3 \%)$ & $31(39,7 \%)$ & 78 \\
T otal & 75 & 61 & 136 \\
\hline
\end{tabular}

$\mathrm{DA}=$ doença arterial $; \mathrm{EP}=$ embolia pulmonar; $\mathrm{TVP}=$ trombose venosa profunda.

A RN I média foi de 2,64 $\pm 0,45$, não havendo diferença estatística entre sexos, diferentes faixas etárias ou tipo de doença de base.

O ito pacientes apresentaram intercorrências não relacionadas ao uso deanticoagulanteoral, não levando a al teração no tratamento anticoagulante (T abela 3).

0 número total de complicações hemorrágicas foi de $21(15,4 \%)$, equivalente a 38,8 por 100 pacientes/ ano, sen do uma considerada grave $(0,73 \%)$, ou 1,9 por 100 pacientes/ano. Asdemaisenquadraram-senascate- gorias moderada e leve. A freqüência e os tipos de hemorragia encontram-se na T abela 4.

$\mathrm{N}$ ão houve diferença estatística quanto à freqüência de hemorragia com relação ao sexo, faixa etária, tipo de doença e dose média de warfarina, porém houve rel ação entre a R N I média e a freqüência de hemorragia ( $T$ abela 5).

U m paciente $(0,7 \%)$, com história anterior de úlcera gastroduodenal, teve episódio hemorrágico (hematêmese), que foi considerado grave, e para o qual

T abela 3 - Intercorrênciasnão relacionadasao uso do anticoagulanteoral do grupo depacientes com doença vascular periférica tratados com antagonista da vitamina $\mathrm{K}$

\begin{tabular}{lcc}
\hline Intercorrência & Freqüência & Percentagem \\
\hline Chagas + bronquite & 1 & 0,7 \\
D iabetes & 1 & 0,7 \\
Edema de membro inferior direito & 1 & 0,7 \\
Edema de membro inferior esquerdo & 2 & 1,5 \\
Sinusite & 1 & 0,7 \\
Pneumonia & 1 & 0,7 \\
D or nas costas & 1 & 0,7 \\
N ega intercorrências & 128 & 94,3 \\
T otal & 136 & $100 \%$ \\
\hline
\end{tabular}


T abela 4 - F reqüência dos tipos de hemorragia e percentagem em relação ao número total de pacientes com doença vascular periférica tratados com antagonista da vitamina $\mathrm{K}$

\begin{tabular}{lccc}
\hline Sangramento & Intercorrência & Freqüência & Percentagem \\
\hline Leve & H ematoma & 4 & 2,9 \\
& Epistaxe & 8 & 5,9 \\
M oderado & Equimose & 6 & 4,4 \\
Grave & H ematúria & 2 & 1,5 \\
& H ematêmese & 1 & 0,7 \\
& Total & 21 & $15,4 \%$ \\
\hline
\end{tabular}

foram necessárias internação e suspen são do anticoagulante. N ão foi necessária, porém, reposição sangüínea. A R I I dessepacienteestava dentro da faixa terapêutica.

$\mathrm{N}$ os pacientes estudados durante o período, nenhum caso de retrombose foi identificado. 0 s edemas encontrados durante 0 seguimento eram residuais e se mantinham desde o quadro agudo de trombose.

U ma paciente teve suspeita de alergia pela warfarina, que, entretanto, foi depois demonstrado ser devida a uso de um antiinflamatório. $\mathrm{N}$ ão foram verificadas outras complicações, como necrose cutânea, alopécia, etc.

\section{D iscussão}

0 principal risco da anticoagulação oral éa hemorragia1,26,27. Em nosso estudo, foram observados $15,4 \%$
(38,8 por 100 pacientes/ano) de eventos hemorrágicos nos 136 pacientes acompanhados, sendo a gran de maioria destes de intensidade leve $(13,2 \%)$ ou moderada $(2,2 \%)$. U m caso apenas $(0,7 \%)$, de hemorragia gastrintestinal, foi considerado grave, para o qual foi necessária internação do paciente.

A incidência de complicações hemorrágicas não é homogênea na literatural,18,27. Para o TEV, a incidência de episódios de sangramento maior varia de 0,4 a $12,8 \%$, sendo a maioria dos dados tirados de trabal hos prospectivos em que a warfarina foi comparada com diferentes tipos e regimes de heparinas de baixo peso molecular18,27,28.

Alguns trabalhos comparam também os resultados e complicações dos AVK usados em período curto e longo após episódio de TEV. M etanálises desses resultados mostraram sangramento maior em

T abela 5 - Relação entreR I média edosemédia da medicação efreqüência de hemorragia em pacientes com doença vascular periférica tratados com antagonista da vitamina $\mathrm{K}$

\begin{tabular}{lcc}
\hline H emorragia & R I média & D ose média \\
\hline Sim & $3,07 \pm 0,36$ & $8,15 \pm 2,80 \mathrm{mg}$ \\
N ão & $2,67 \pm 0,45$ & $8,38 \pm 2,75 \mathrm{mg}$ \\
P & 0,0002 & 0,75 \\
\hline
\end{tabular}

$\mathrm{RNI}=$ razão normatizada internacional . 
$0,3 \%$ nos pacientes tratad os a curto prazo e $2,5 \%$ nos pacientes com tratamento longo 29 . Em estudo semeIhante ao nosso, Poli et al., acompanhando 903 pacientes por um período 1,8 anos, encontraram uma freqüência de hemorragia total, grave e fatal de 5, 1,1 e 0,06 por 100 pacientes/ano, respectivamente. $G$ reen et al. ${ }^{26}$, em auditoria de duas clínicas de anticoagulação no Reino U nido, incluindo 7.400 pacientes, encontraram, em 10 meses, uma incidência de hemorragia total de $2,9 \%$ e maior de $0,72 \%$. Palareti et al. ${ }^{30}$, em estudo realizado em 34 centros na I tália, com mais de 2.000 pacientes, encontraram uma incidência total de 7,6 casos de hemorragia por pacientes/ano, sendo 0,25 fatais e 1,1 considerados maiores.

Em nosso meio, em dois levantamentos retrospectivos prévios em nosso ambulatório, 2,6 e 1,5\% dos doentes tiveram seu tratamento suspenso por hemorragia ${ }^{13,24}$. Lourenço et al. ${ }^{25}$, em trabalho realizado no Ambulatório de Anticoagulação da U niversidadeFederal deSão Paulo (U N IFESP), referiram a ocorrência de sangramento de $0,3 \%$ em 4.600 consultas, sendo a maioria considerada sem maior gravidade.

É possível que, no trabalho atual, prospectivo, o número maior de hemorragias não-graves se deva ao interrogatório minucioso realizado por uma só investigadora, que levou os pacientes a se lembrarem de pequenas equimoses esangramentosnasais, queteriam, de outra maneira, passado desapercebidos. Esse fato sugere que os sangramentos considerados moderados no presente trabalho estão em número muito próximo aos citados na literatura como sangramento total. 0 utros autores, em trabalhos prospectivos, referem também alta incidência de hemorragia leve, de até de $23 \% 18,31$. Alguns trabalhos e metanálises nem sequer citam a freqüência de sangramentos menores ${ }^{32}$.

É preciso lembrar, também, que enquanto nosso estudo selimitou a pacientescom TEV edoença arterial periférica, os resultados descritos na maioria das clínicas de anticoagulação incluem principalmente pacientes cardiopatas, podendo esses pacientes ter o comportamento diferente em relação à anticoagulação oral.

0 utro aspecto a considerar é que os pacientes com doença arterial periférica são bastante heterogêneos e, freqüentemente, portadores de outras afecções, como hipertensão arterial e diabetes. Raros são os trabalhos que enfocaram esse tipo de paciente com relação ao tratamento anticoagulante ${ }^{23}$; a maioria são estudos comparativos entre AVK e antiplaquetários em enxer- tos distais nos membros inferiores,33-35. Em trabalho anterior, em nosso ambulatório de anticoagulação, estudamos retrospectivamente 230 pacientes com doença arterial periférica, tratados com warfarina, num período de 17 anos. A freqüência total de sangramento nesses pacientes foi de $29 \%$, sendo que grande hemorragia ocorreu em $1,7 \%$ dos $\operatorname{casos}^{36}$. N o presente trabaIho, a incidência total de sangramento em doentes arteriaisfoi semelhante, de $21,6 \%$, sem casos de sangramento grave.

U m aspecto que tem sido discutido por vários autores é de não haver uma uniformidade nos critérios dedefinição dosgraus dehemorragia ${ }^{18,27,37}$. A maioria dos autores divideo sangramento em maior emenor ${ }^{20}$. Em nosso estudo, classificamos como graves os casos descritos na literatura como maiorese, assim, classificamos o paciente com hemorragia gastrintestinal. C riamos, entretanto, uma subdivisão para os demaissangramentos em moderados e leves, para tentar descrever melhor estes episódios.

$\mathrm{N}$ os doentes por nós acompanhados, não ocorreram casos de hemorragia intracraniana ou morte em conseqüência de hemorragia. Em pacientes tratados por TEV com warfarina, em ensaios clínicos controlados randomizados, a mortalidade também foi baixa, próxima a zero na maioria dos trabal hos ${ }^{18,38}$. M etanálise realizada por Linkins et al. ${ }^{27}$, reunindo 10.757 pacientes (4.374 pacientes/ano) tratadoscom AVK por TEV, em ensaios randomizados ou coortes prospectiVos, mostrou uma incidência de 1,15 casos por 100 pacientes/ano de hemorragia intracraniana. Essa incidência parece ser maior ainda em pacientes com fibrilação atrial, infarto do miocárdio, válvulas prostéticas cardíacas e acidente vascular encefálico, onde tem variado de 0,2 a 2,9\%39-42.

$D$ as variáveis estudadas, apenas o nível médio de RN I foi estatisticamente diferente entre os pacientes com e sem sangramento, sendo maior nos primeiros. Relação entreintensidade da anticoagulação efreqüência de sangramento tem sido descrita em trabalhos realizados com pacientes tratados por diferentes doenças 26,43-47. Embora, no presentetrabal ho, tenha existido relação entre nível de RN I e sangramento, não foi encontrada relação entre a dose média de warfarina e sangramento, indicando que, embora doses excessivas de AVK possam aumentar os níveis de anticoagulação, na população estudada, este não foi um fator determinante, dependendo, talvez, mais das características individuais de cada paciente ${ }^{48}$. 
N o presente trabalho, não houve relação entre a freqüência de sangramento e o sexo ou idade. Resultados similares foram encontrados por Poli et al. ${ }^{17}$, estudando um número maior de pacientes. Trabal hos antigos da literatura já indicavam essa falta de relação entresangramento esexo $0^{49-51}$; outros, entretanto, indicam uma freqüência maior de hemorragias nas mulheres em uso dos AVK 37,52 .

Com relação à idade, vários autores, como nós, não encontraram relação idade/sangramento 37,53-55; entretanto, outrosindicam ser um fator independente de sangramento em pacientes tratados com AVK, principalmentecom idadesuperior a 75 anos ${ }^{47,56,57}$. V ários estudos, principalmente em fibrilação atrial, sugerem que, após essa idade, étambém mais comum o sangramento intracraniano, quando há aumento de RN I 44,58

Com relação a complicações não-hemorrágicas, com exceção de um paciente que apresentou reação alérgica, posteriormente demonstrada como não causada pela warfarina, nenhum caso foi detectado na presente casuística. D essas complicações, a mais séria é a necrose de pele, a qual, embora já tenhamos observado em pacientes tratados em nosso ambulatório, não foi verificada na presente casuística, talvez por sua baixa freqüência ${ }^{21}$. $N$ ão verificamos casos de recidiva de TEV ou arterial. Como nossos casos foram sorteados, para representar a população total de nossos doentes, é provável que controle na anticoagulação, quemantevea RN I dentro da faixa proposta, em torno de $70 \%$ do tempo, tenha sido eficiente, necessitando-se um número maior de pacientes para ser detectado. T rabal hos prospectivos comparando a warfarina com outros anticoagulantes têm mostrado recidiva clínica de tromboembolismo venoso, variando de 2 a $4 \%$ com warfarina 59,60 .

0 AAFM B atende principalmente pacientes de baixo nível socioeconômico efreqüentementemoradores de fora do município de Botucatu, situação esta freqüentementeapontadacomo causa dedificuldadede utilização de tratamento com AVK. O s resultados do presente trabalho, principalmente com relação à freqüência de sangramentos graves, são, de modo geral, semel hantes aos publicados na literatura internacional, em estudos realizados em clínicas de anticoagulação, o que confirma a viabilidade do tratamento anticoagulantecom warfarina em nosso meio, mesmo em pacientes de baixo nível socioeconômico, desde que realizado em ambulatórios especializados.

\section{R eferências}

1. Ansell J, H irsh J, Poller L, Bussey H, Jacobson A, H ylek E. The pharmacology and management of the vitamin $K$ antagonists: theSeventh ACCP C onferenceon Antithrombotic and Thrombolytic Therapy. Chest. 2004;126:204S-33S.

2. Schulman S. Clinical practice. $C$ are of patients receiving longterm anticoagulant therapy. N Engl J M ed. 2003;349:675-83.

3. Cortelazzo S, Finazzi G, Viero $P$, et al. Thrombotic and hemorrhagic complications in patients with mechanical heart valve prosthesis attending an anticoagulation clinic. Thromb H aemost. 1993;69:316-20.

4. European/Australasian Stroke Prevention in Reversible Ischemia Trial (ESPRIT). O ral anticoagulation in patients after cerebral ischemia of arterial origin and risk of intracranial hemorrhage. Stroke. 2003;34:e45-6.

5. Sarac T P, H uber T S, Back M R, et al. W arfarin improves the outcome of infrainguinal vein bypass grafting at high risk for failure. J V asc Surg. 1998;28:446-57.

6. Aithal GP, D ay CP, Kesteven PJ, D aly AK. Association of polymorphisms in the cytochrome P450 CYP2C 9 with warfarin doserequirement and risk of bleeding complications. Lancet. 1999;353:717-9.

7. H igashi M K, V eenstra DL, Kondo LM, et al. Association between CYP2C 9 gen etic variants and anticoagulation-related outcomesduring warfarin therapy. JAM A. 2002;287:1690-8.

8. Majerus PW, Broze GJ, Miletich JP, Tollefsen DM. Anticoagulant, thrombolytic, and antiplatelet drugs. In: $H$ ardman J G, Limbird LE, M olinoff PB, Ruddon RW, Gilman $A G$, editors. Goodman \& G ilman's thepharmacological basis of therapeutics. 9th ed. N ew York: M cGraw-Hill; 1996. p. 1341-59.

9. M annucci PM. Genetic control of anticoagulation. Lancet. 1999;353:688-9.

10. O 'Reilly RA, Aggeler PM, H oag M S, Leong LS, Kropatkin $M L$. H ereditary transmission of exceptional resistance to coumarin anticoagulant drugs. The first reported kindred. N Engl J M ed. 1964;271:809-15.

11. Penning-van Beest FJ, G eleijnse JM, van M eegen $E$, V ermeer C, Rosendaal FR, Stricker BH . L ifestyleand diet as risk factors for overanticoagulation. J Clin Epidemiol. 2002;55:411-7.

12. Loeliger EA. Laboratory control optimal therapeutic ranges and therapeutic quality control in oral anticoagulants. Acta H aematol. 1985;74:125-31.

13. Thomazini I A, M affei FH, Yuan FC, Zannini JM , LastóriaS, $\mathrm{N}$ oguerira FL. T erapêutica anticoagulante oral a longo prazo em trombose venosa profunda: é possível em pacientes de baixo nível socioeconômico nos paises em desenvolvimento? Rev I bero AmericanaT rombose eH emostasia. 1995;8:201-5.

14. M clnnes GT, H elenglass $G$. The performance of clinics for outpatient control of anticoagulation. J R Coll Physicians Lond. 1987;21:42-5.

15. ÁngelesF ernandez M. G estión del tratamiento anticoagulante oral: unidad centralizada. Hematologica. 2001;86(Suppl 2):117.

16. Berrettini M , Agnelli G. M anagement of oral anticoagulant in I taly. Semin Thromb H emost. 1999;25:27-31.

17. Poli $D$, Antonucci $E$, Lombardi A, et al. Low rate of bleeding and thrombotic complications of oral anticoagulant therapy independent of age in the real - practice of an anticoagulation clinic. Blood Coagul Fibrinolysis. 2003;14:269-75. 
18. Levine M N, Raskob G, Beyth RJ, Kearon C, Schulman S. $\mathrm{H}$ emorrhagic complications of anticoagulant treatment: the Seventh ACCP Conference on Antithrombotic and Thrombolytic Therapy. Chest. 2004;126:287S-310S.

19. Kucher N, Connolly S, Beckman JA, et al. International normalized ratio increase before warfarin - associated hemorrhage: brief and subtle. Arch Intern M ed. 2004;164: 2176-9.

20. $H$ arenberg J, H offmann $U, H$ uhle G, W inkler $M$, Bayerl C. Cutaneous reactions to anticoagulants: recognition and management. Am J Clin D ermatol. 2001;2:69-75.

21. Ad-EI DD, M eirovitz $A, W$ einberg $A$, et al. W arfarin skin necrosis: local and systemic factors. Br J Plast Surg. 2000;53: 624-6.

22. Buller HR, Sohne M, M iddeldorp S. T reatment of venous thromboembolism. J Thromb H aemost. 2005;3:1554-60.

23. Timi JRR, M oraes LM, Góes Junior DCA, M artins $M$, M oreira RCR, Abrão E. Riscos do uso do anticoagulante oral após embolectomia arterial. Rev Bras M ed. 1988;45:316-7.

24. M affei FH A, T homazini IA, Yuan FC, et al. A companhamento da terapêutica anticoagulante em ambulatório especializado. Cir V asc Ang. 1988;4:7-13.

25. Lourenço DM, M orelli VM, Vignal CV. Tratamento da superdosagem de anticoagulantes orais. Arq Bras Cardiol. 1998;70:9-13.

26. Rhodes $S, G$ reen $E S$, Bond $S$, et al. Bleeding complications of oral anticoagulant therapy: a prospectiveaudit. $\mathrm{Br}] \mathrm{H}$ aematol. 2004;125 Suppl 1:57.

27. Linkins LA, Choi PT, D ouketis JD. Clinical impact of bleeding in patients taking oral anticoagulant therapy for venous thromboembolism: a meta-analysis. Ann Intern M ed. 2003;139:893-900.

28. van der $H$ eijden JF, $H$ utten $B A$, Büller $H R$, Prins $M H$. $\mathrm{V}$ itamin $\mathrm{K}$ antagonists or low-molecular-weight heparin for the long term treatment of symptomatic venous thromboembolism (CochraneR eview). T heC ochraneL ibrary. 2006;3:CD 001367

29. H utten $B A$, Prins $M H$. H utten $B A$, Prins M H . D uration of treatment with vitamin $\mathrm{K}$ antagonistsin symptomatic venous thromboembolism (Cochrane Review). In: The Cochrane Library. Oxford: U pdate Software. 2006.

30. Palareti G, Leali N, Coccheri S, et al. Bleeding complications of oral anticoagulant treatment: an inception-cohort, prospective collaborative study (ISCOAT) I talian Study on Complications of $O$ ral Anticoagulant Therapy. Lancet. 1996;348:423-8.

31. M ohr JP, Thompson JL, Lazar RM, et al. A comparison of warfarin and aspirin for prevention of recurrent ischemic stroke. N Engl J M ed. 2001;345:1444-51.

32. van $W$ al raven $C, H$ art RG , Singer $D E$, etal. $O$ ral anticoagulants vs. aspirin in nonvalvular atrial fibrillation: an individual patient meta-analysis. JAM A. 2002;288:2441-8.

33. K retschmer $G, H$ erbst $F$, Prager $M$, et al. A decade of oral anticoagulant treatment to maintain autologousvein graftsfor femoropopliteal atherosclerosis. Arch Surg. 1992;127:1112-5.

34. Johnson W C, W illiford W O ; D epartment of V eteransAffairs Cooperative Study \#362. Benefits, morbidity, and mortality associated with long-term administration of oral anticoagulant therapy to patients with peripheral arterial bypass procedures: aprospectiverandomized study. J V asc Surg. 2002;35:413-21.
35. T angelder M J, Algra A, Lawson JA, H ennekes S, Eikelboom $B C$. O ptimal oral anticoagulant intensity to prevent secondary ischemic and hemorrhagic eventsin patients after infrainguinal bypass graft surgery. D utch BO A Study Group. J V asc Surg. 2001;33:522-7.

36. SantosFC, M enegon FTF, Thomazini-Santos IA, G ianini M , Lastória S, M affei FH A. Terapia anticoagulantealongo prazo em doença arterial periférica. J Vasc Bras. 2003;2:228.

37. Finn $S D, M C D$ onell $M, M$ artin $D$, et al. Risk factors for complications of chronic anticoagulation. A multicenter study. W arfarin 0 ptimized O utpatient Follow-up Study Group. Ann Intern M ed. 1993;118:511-20.

38. Landefeld C S, Beyth RJ. Anticoagulant-Related Bleeding: clinical epidemiology, prediction, and prevention. Am J M ed. 1993;95:315-28.

39. Albers GW, Amarenco P, Easton JD, Sacco RL, Teal P. Antithromboticand thrombolytic therapy for ischemic stroke: the Seventh ACCP Conference on Antithrombotic and Thrombolytic Therapy. Chest. 2004;126:483S-512S.

40. $H$ arrington RA, Becker RC, Ezekowitz $M$, etal. Antithrombotic therapy for coronary artery disease: the Seventh ACCP Conference on Antithrombotic and Thrombolytic Therapy. Chest. 2004;126:513S-48S.

41. Salem D N, Stein PD, Al-Ahmad A, et al. Antithrombotic therapy in valvular heart disease native and prosthetic: the Seventh ACCP Conference on Antithrombotic and Thrombolytic Therapy. Chest. 2004;126:457S-82S.

42. Singer DE, Albers GW, Dalen JE, Go AS, Halperin JL, $M$ anning W J. Antithrombotic Therapy in Atrial Fibrillation: the Seventh ACCP Conference on Antithrombotic and Thrombolytic Therapy. Chest. 2004;126:429S-56S.

43. H ylek EM , Singer D E. Risk factorsfor intracranial hemorrhage in outpatients taking warfarin. Ann Intern M ed. 1994;120: 897-902.

44. Secondary prevention in non-rheumatic atrial fibrillation after transient ischaemic attack or minor stroke. EAFT (European Atrial Fibrillation Trial) Study Group. Lancet. 1993;342:1255-62.

45. Cannegieter $S C$, Rosendaal FR, W intzen $A R$, van der M eer FJ, Vandenbroucke JP, B riet E. O ptimal oral anticoagulant therapy in patients with mechanical heart valves. N Engl J M ed. 1995;333:11-7.

46. H ull R, Hirsh J, Jay R, et al. Different intensities of oral anticoagulant therapy in the treatment of proximal-vein thrombosis. N Engl J M ed. 1982;307:1676-81.

47. Crowther M A, G insberg JS, Julian J, et al. A comparison of two intensities of warfarin for the prevention of recurrent thrombosis in patients with antiphospholipid antibody syndrome. N Engl J M ed. 2003;349:1133-8.

48. Torn $M, V$ an der $M$ eer FJ, Rosendaal FR. Lowering the intensity of oral anticoagulant therapy: effects on the risk of hemorrhage and thromboembolism. Arch Intern M ed. 2004;164:668-73.

49. Coon WW, W illis PW 3rd. H emorrhagic complications of anticoagulant therapy. Arch Intern M ed. 1974;133:386-92.

50. D avis $F B$, Estruch $M T$, Samson-Corvera EB, Voigt GC, Tobin JD. M anagement of anticoagulation in outpatients: experience with an anticoagulation service in a municipal hospital setting. Arch Intern M ed. 1977;137:197-202. 
51. Palareti G, H irsh J, Legnani C, et al. O ral anticoagulation treatment in the elderly. Arch Intern M ed. 2000;160:470-8.

52. V an der M eer FJ, R osendaal FR, V andenbroucke JP, Briet E. Bleeding complications in oral anticoagulant therapy: analysis of risk factors. Arch Intern M ed. 1993;153:1557-62.

53. Risksof long-term oral anticoagulant therapy in elderly patients after myocardial infarction. Second report of the Sixty Plus Reinfarction Study Research G roup. Lancet. 1982;1:64-8.

54. W ickramasinghe LS, Basu SK, Bansal SK. Long-term oral anticoagulant therapy in elderly patients. Age Ageing. 1988;17:388-96.

55. Joglekar M, M ohanaruban K, Bayer AJ, Pathy M S. C an old people on oral anticoagulants be safely managed as outpatients? Postgrad M ed J. 1988;64:775-7.

56. Errichetti AM, Holden A, Ansell J. M anagement of oral anticoagulant therapy: experience with an anticoagulation clinic. Arch Intern M ed. 1984;144:1966-8.

57. Petty GW, Lennihan $L, M$ ohr JP, et al. Complications of long-term anticoagulation. Ann N eurol. 1988;23:570-4.

58. Bleeding during antithrombotic therapy in patientswith atrial fibrillation. The Stroke Prevention in Atrial Fibrillation Investigators. Arch Intern M ed. 1996;156:409-16.
59. Pini $M$, Aiello S, M anotti $C$, et al. Low molecular weight heparin versus warfarin in the prevention of recurrences after deep vein thrombosis. Thromb H aemost. 1994;72:191-7.

60. D asSK, C ohen AT, Edmondson RA, M elissari E, Kakkar VV. Low-molecular-weight heparin versus warfarin in the prevention of recurrent venous thromboembolism: a randomized trial. W orld J Surg. 1996;20:521-6; discussion 526-7.

\section{Correspondência:}

Francisco H. de A. M affei

Rua Eng. Edgar Egidio de Sousa, 303/51

CEP 01233-020 - São Paulo, SP

Tel./Fax: (11) 3667.7626

E-mail:fhmaffei@uol.com.br 